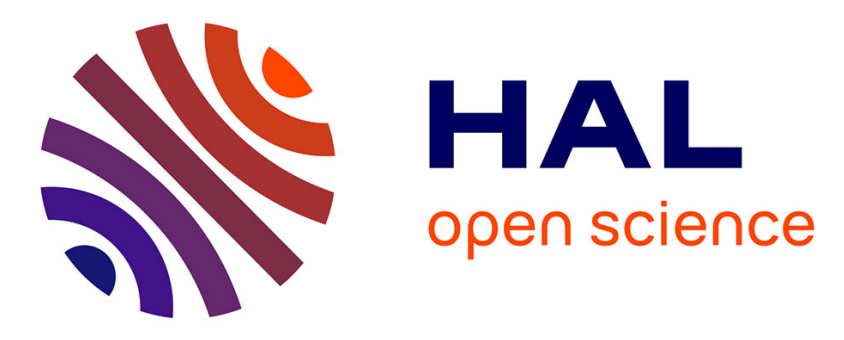

\title{
Statistical Analysis and Directional Coding of Layer-based HDR Image Coding Residue
}

\author{
Kutan Feyiz, Fatih Kamisli, Emin Zerman, Giuseppe Valenzise, Alper Koz,
} Frederic Dufaux

\section{- To cite this version:}

Kutan Feyiz, Fatih Kamisli, Emin Zerman, Giuseppe Valenzise, Alper Koz, et al.. Statistical Analysis and Directional Coding of Layer-based HDR Image Coding Residue. 19th International Workshop on Multimedia Signal Processing (MMSP), Oct 2017, London-Luton, United Kingdom. 10.1109/mmsp.2017.8122244 . hal-01567379

\section{HAL Id: hal-01567379 \\ https://hal.science/hal-01567379}

Submitted on 10 Jan 2020

HAL is a multi-disciplinary open access archive for the deposit and dissemination of scientific research documents, whether they are published or not. The documents may come from teaching and research institutions in France or abroad, or from public or private research centers.
L'archive ouverte pluridisciplinaire HAL, est destinée au dépôt et à la diffusion de documents scientifiques de niveau recherche, publiés ou non, émanant des établissements d'enseignement et de recherche français ou étrangers, des laboratoires publics ou privés. 


\section{Statistical Analysis and Directional Coding of Layer- based HDR Image Coding Residue}

\author{
Kutan Feyiz \\ Center for Image Analysis \\ Electrical and Electronics Eng. \\ Middle East Technical University \\ Ankara, Turkey \\ kutan.feyiz@metu.edu.tr
}

\author{
Fatih Kamisli \\ Center for Image Analysis \\ Electrical and Electronics Eng. \\ Middle East Technical University \\ Ankara, Turkey \\ kamisli@metu.edu.tr
}

Alper Koz

Center for Image Analysis

Middle East Technical University

Ankara, Turkey

koz@metu.edu.tr

\author{
Emin Zerman \\ LTCI, Télécom ParisTech, \\ Université Paris-Saclay, \\ 75013 Paris, France \\ emin.zerman@telecom- \\ paristech.fr
}

\author{
Giuseppe Valenzise \\ Laboratory des Signaux et \\ Systems \\ CNRS-Centrale Supelec- \\ Universite Paris-Sud \\ giuseppe.valensize@12s.centrales \\ upelec.fr
}

\author{
Frederic Dufaux \\ Laboratory des Signaux et \\ Systems \\ CNRS-Centrale Supelec- \\ Universite Paris-Sud \\ frederic.dufaux@12s.centralesupe \\ lec.fr
}

\begin{abstract}
Existing methods for layer-based backward compatible high dynamic range (HDR) image and video coding mostly focus on the rate-distortion optimization of base layer while neglecting the encoding of the residue signal in the enhancement layer. Although some recent studies handle residue coding by designing function based fixed global mapping curves for 8-bit conversion and exploiting standard codecs on the resulting 8-bit images, they do not take the local characteristics of residue blocks into account. Inspired by the local anisotropic characteristics of the residue signal and directional methods for motion compensated low dynamic range (LDR) video coding, in this paper we first investigate whether HDR image coding residue exhibits also local anisotropic characteristics. Specifically, we verify directional structures in residue blocks by means of auto-covariance analysis for different bitrates, spatial activities and dynamic ranges as the main variables in HDR image coding. Then, we compare the rate distortion performances of directional coding methods with the baseline residue coding methods in the literature along with different combinations of 8-bit conversion methods. The experiments indicate that content dependent 8-bit conversions and directional coding significantly outperforms the existing function based 8-bit conversions and typical coding for residue coding.
\end{abstract}

Keywords- High Dynamic Range, residue coding, local characteristics, auto-covariance analysis, directional coding

\section{INTRODUCTION}

A major challenge for HDR imaging is an efficient compression scheme due to the higher storage size of HDR images represented in floating points compared to 8-bit LDR representation. Backward compatibility to the existing 8-bit displays for such a compression scheme is an essential requirement for the wide acceptance of this technology by the users [1]-[3].
The existing methods proposed for this purpose can be categorized in two classes based on two different application scenarios. In the first class, the LDR video is produced from the HDR video by applying local artistic effects changing with respect to time and spatial position as in the post production of film making. As the LDR video is produced after a tidy post processing stage in this case, the quality of the LDR video is preserved during encoding and the corresponding HDR video is estimated from the given LDR video by an inter-layer prediction [4]. The residue obtained as the difference between the HDR video and its estimation is encoded and added to the LDR video stream. The interlayer prediction is achieved by a block-wise approach in [5]-[6]. A block from an HDR video frame is approximated by applying a linear multiplication and an offset addition to the corresponding LDR block. Instead of block-wise prediction, some other approaches also adopt frame-wise estimation of HDR frames by LDR frames [4].

In the second class of methods [3], [7]-[9], the LDR video is instead produced from HDR by a global tone mapping operation before coding (Fig. 1). Such a strategy is mainly applicable for broadcasting of HDR video. The LDR video goes through the standard video encoding and decoding. Then, the HDR video is reconstructed from LDR video by applying an inverse tone mapping. Mai et al. [3] derive a solution for optimum tone mapping which minimizes the mean-square-error (MSE) between the original and reconstructed HDR video. Their solution is based on a statistical model, which approximates the distortion in the sequence of tone-mapping, encoding, decoding, and inversetone mapping. This solution is improved in [7] by using perceptually uniform luminance values instead of the logarithmic values. Recent studies further explore the optimization of tone mapping by considering the rate of the base and enhancement layers together [8]. In [9], this method 
which independently performs tone mapping for each frame is replaced with joint tone mapping of successive video frames.

While these approaches mainly focus on the rate-distortion optimization of base layer, the encoding of the residue in the enhancement layer has received less attention. Although some recent studies [10], [11] design function based global mapping curves for the 8-bit conversion and uses typical codecs for residue coding, they do not consider the local characteristics of residue blocks for encoding. However, in well-studied motion compensated LDR video coding [12], [14], it is verified that the residue blocks exhibit local anisotropic characteristics, which are more efficiently compressed with directional transforms than the traditional 2-D DCT.

In this paper, we first investigate whether HDR image coding residue also exhibits local anisotropic characteristics by means of performing auto-covariance analysis on residue blocks for different bitrate, spatial activity and dynamic range as the main variables in HDR image coding. After the verification of directional characteristics on HDR image coding residue, we examine the rate distortion performances of content based 8-bit conversion and directional coding methods compared to a recent baseline method performing function based 8-bit conversion and typical coding.

In the rest of this paper, Section 2 presents the general scheme of the proposed two layer HDR image/video coding with the baseline and residue layers. Section 3 gives an overview of auto-covariance analysis performed on residue blocks and the experimental results for the verification of directional characteristics on HDR image coding residue. Section 4 gives the details of proposed residue coding with the utilized 8-bit conversions and directional coding and the ratedistortion curves in comparison with the baseline schemes. Finally, conclusions are presented in Section 5.

\section{General Scheme of the Proposed Two Layer Coding}

Fig. 1 illustrates the general scheme for backward compatible HDR image compression with the baseline and residue layers [7]. Firstly, after RGB to LogLuv color space transformation, HDR frame/image is mapped into 8-bit LDR image by using a tone mapping operator. The LDR image goes through the standard encoding and decoding. Then, HDR image is reconstructed from the decoded LDR image by applying an inverse tone mapping and the residue frame $\left(r_{n}\right)$ is obtained as the difference of the original and reconstructed HDR images. We use the optimum (inverse) tone mapping derived by Mai et al. [3]. However, other tone mappings could also be utilized without loss of generality. As an essential initial stage, we first investigate whether the obtained residue frames $\left(r_{n}\right)$ exhibit directional characteristics or not by performing auto-covariance analysis in the next section.

The obtained residue frame, which is in floating point form, is converted to 8-bits. While the baseline method in the literature adopts a function based fixed mapping to perform this conversion, we also examine the performance of content based solutions by adapting the optimum tone mapping of Mai et al. [3] for 8-bit conversion (Section IV.A). The resulting 8bit images go through standard [11] or directional coding as explained in (Section IV.B).

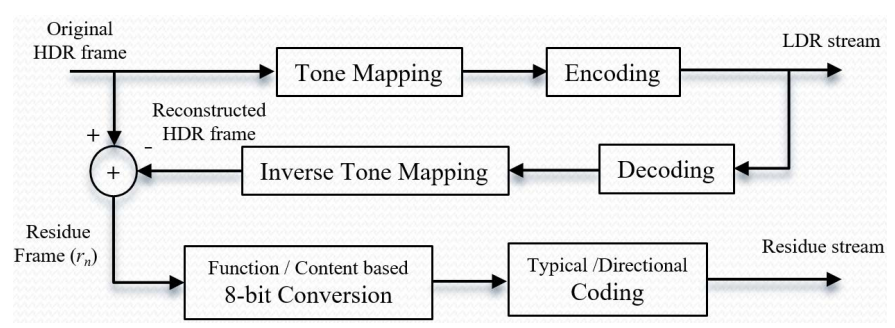

Fig. 1 General scheme for layer based backward compatible HDR image/video compression [7]

The decoder both receives the LDR stream and residue stream. The LDR stream is decoded and inverse tone mapped. The residue stream is also decoded and the inverse of the 8-bit conversion is applied to the decoded signal to get the reconstructed residue frame. Then, the inverse tone mapped HDR frame and the reconstructed residue frame added to get the reconstructed HDR frame in the decoder side. During the rate-distortion tests, the rate is taken as the total bit rate of LDR base layer and residue layer together and the distortion is computed between the original and reconstructed HDR frames.

\section{Auto-COVARIANCE ANALysis OF THE RESIDUE FrameS}

This section presents empirical and statistical analyses of layer based backward compatible HDR image coding residuals. The analysis methods are similar to those used for analyzing motion-compensated prediction residuals in [12]. In particular, the characteristics of the auto-covariance of local regions of HDR coding residuals are analyzed to conclude whether directional coding methods can be applied for residue coding.

\section{A. Utilized Auto-Covariance Models}

To quantify the results of the statistical analysis of HDR coding residuals, the auto-covariance of 8x8-pixel blocks of residue frame $\left(r_{n}\right)$ are modeled with the auto-covariance of a 2-D Markov process. We use two auto-covariance models that were proposed in [12] for similar statistical analysis. The first is a separable auto-covariance model given by

$$
R_{S}(I, J)=\rho_{1}^{|I|} \rho_{2}|J|,
$$

where $\rho_{1}$ is the correlation parameter across the horizontal or vertical direction, and $\rho_{2}$ that along the other direction. The larger correlation coefficient can always be chosen as $\rho_{1}$ for convenience. Since this is a separable model, it favors horizontal or vertical directions and cannot model well the statistical characteristics of residual blocks with strong correlation along other directions such as diagonal directions. Therefore, another auto-covariance model shown below in Equation 2 is also used in the statistical analysis,

$$
R_{g}(\theta, I, J)=\rho_{1}^{|I \cos (\theta)+J \sin (\theta)|} \rho_{2}^{|-I \sin (\theta)+J \cos (\theta)|} .
$$

It is referred to as the generalized model since it generalizes the separable model by introducing an additional parameter $\theta$, allowing rotation of the horizontal and vertical axes of the 


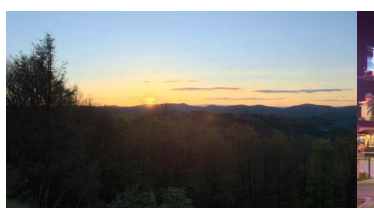

(a) "AirBellowsGap"

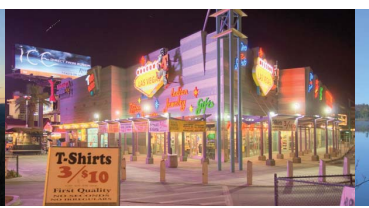

(b) "LasVegasStore"

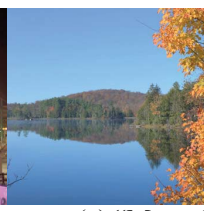

(c) "MasonLake(1)"

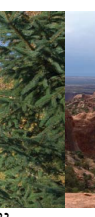

$\pm$ (d) "RedwoodSunset"

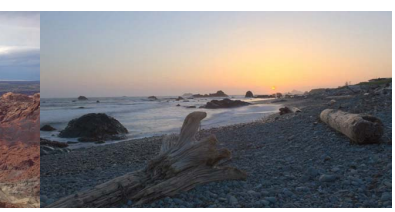

Fig. 2 The selected HDR image set (tone mapped versions) for the experiments separable model. Hence, the generalized model can better adapt and model the statistical characteristics of residual blocks which have strong correlation along directions other than horizontal and vertical.

Each 8x8-pixel block of the HDR coding residual frames is modeled with both the separable and the generalized autocovariance model by estimating parameters $\rho_{1}$ and $\rho_{2}$ (and $\theta$ for the generalized model) so that the parametric autocovariance models provide the best fit to the actual autocovariance of each $8 \times 8$-pixel block. This is done by first estimating the actual auto-covariance of each residual block using the unbiased correlation estimator and then using the optimization toolbox of Matlab to find the best parameters $\rho_{1}$ and $\rho_{2}$ (and $\theta$ for the generalized model) so that the separable and generalized auto-covariance models fit the estimated unbiased auto-correlation in the least square sense.

The estimated $\rho_{1}$ and $\rho_{2}$ pairs from all $8 \times 8$ residual blocks can then be plotted as scatter plots for each residual frame to be analyzed using both the separable and generalized autocovariance models. Each point in the scatter plots represents the estimated $\left(\rho_{1}, \rho_{2}\right)$ pair from one 8x8-pixel residual block. In this way, statistical characteristics of HDR coding residuals can be evaluated, as it is done in the next section for several HDR coding residue frames.

\section{B. Auto-Covariance Analysis Results}

Five images (32-bit RGBE images as .hdr files), which exhibit different characteristics in terms of dynamic range and spatial activity, are selected from various HDR image database for the experiments [15]. The tone mapped versions of the selected images are shown in Fig. 2 with their dynamic range and spatial activity in Fig. 3 (a) and (b). The dynamic range is defined as the ratio of the maximum luminance to the minimum luminance in the image. The spatial activity is measured as the standard deviation of the output of a Sobel operator applied to the image. The dynamic range of the images varies from 1.5 to 6 . The spatial activity is in the range of 0.017 to 0.03 . We assume that the selected images coarsely cover the possible dynamic and spatial activity ranges and are sufficiently diverse and representative to perform comparisons. All images are in size 1920x1080 and inspected not to possess any typical HDR acquisition artifact [15].

The selected images undergo tone mapping, coding, decoding and inverse tone mapping and the residue images are obtained as discussed in Section II. The encoding and decoding of LDR images is performed by using JM H.264/AVC reference software [16] with 5 different quantization parameters selected as $\mathrm{QP}=17,22,27,32,37$.
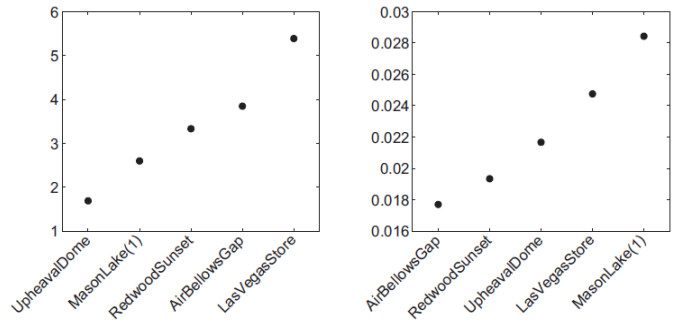

Fig. 3 Dynamic range (left) and spatial activity (right) of the selected HDR image set for experiments [15]

The auto-covariance analysis is performed on $8 \times 8$ blocks as a commonly used block size in compression. The DC mean of each block is subtracted and only the blocks which have sufficient AC energy are considered for the analysis by comparing with a threshold, as the blocks with lower AC energy are typically not coded in compression. The analysis results are given only for the generalized model due to the lack of space.

The first experiment is performed to reveal the effect of bitrate (or QP). Fig. 4 (a), (b) and (c) illustrate the residue images, the scatter plots of the estimates of the correlation coefficients $\left(\rho_{1}, \rho_{2}\right)$, and the histogram of the estimated angles for the generalized auto-covariance model for different QPs for LasVegasStore. Each point in the plots corresponds to the estimate of correlation coefficients $\left(\rho_{1}, \rho_{2}\right)$ in auto-covariance model in Equation (2) for one 8x8 block. As observed, $\rho_{2}$ is mostly smaller than 0.5 and the points are accumulated on the direction of $\rho_{1}$. Combination of large $\rho_{1}$ and small $\rho_{2}$ indicates that a structure exists along the direction of $\rho_{1}$, which implies one dimensional characteristics of the residue blocks. While the quantization increases, the number of blocks surpassing the AC energy threshold also increases due to the higher coding error. As a consequence, the points on the plots show also a scattering behavior towards higher $\rho_{2}$ values for higher QP. The 1D structure of the residue is also becoming more salient for higher quantization as can be observed also on the residue images and histogram of estimated angles $(\theta)$ with higher concentrations at the angles $0^{\circ}, 90^{\circ}$ and $180^{\circ}$.

In order to draw more generic conclusions for varying dynamic range and spatial activity, we also perform the autocovariance analysis on the given HDR image set, while keeping the quantization parameter fixed $(\mathrm{QP}=27)$. Fig. 5 illustrates the scatter plots of the estimates of the correlation coefficients with generalized auto-covariance model for different images. For all the scatter plots, the points are mostly accumulated on the direction of $\rho_{1}$, which exhibits one dimensional characteristics of the residue blocks.

This can also be observed with very low $\rho_{2}$ values for the 

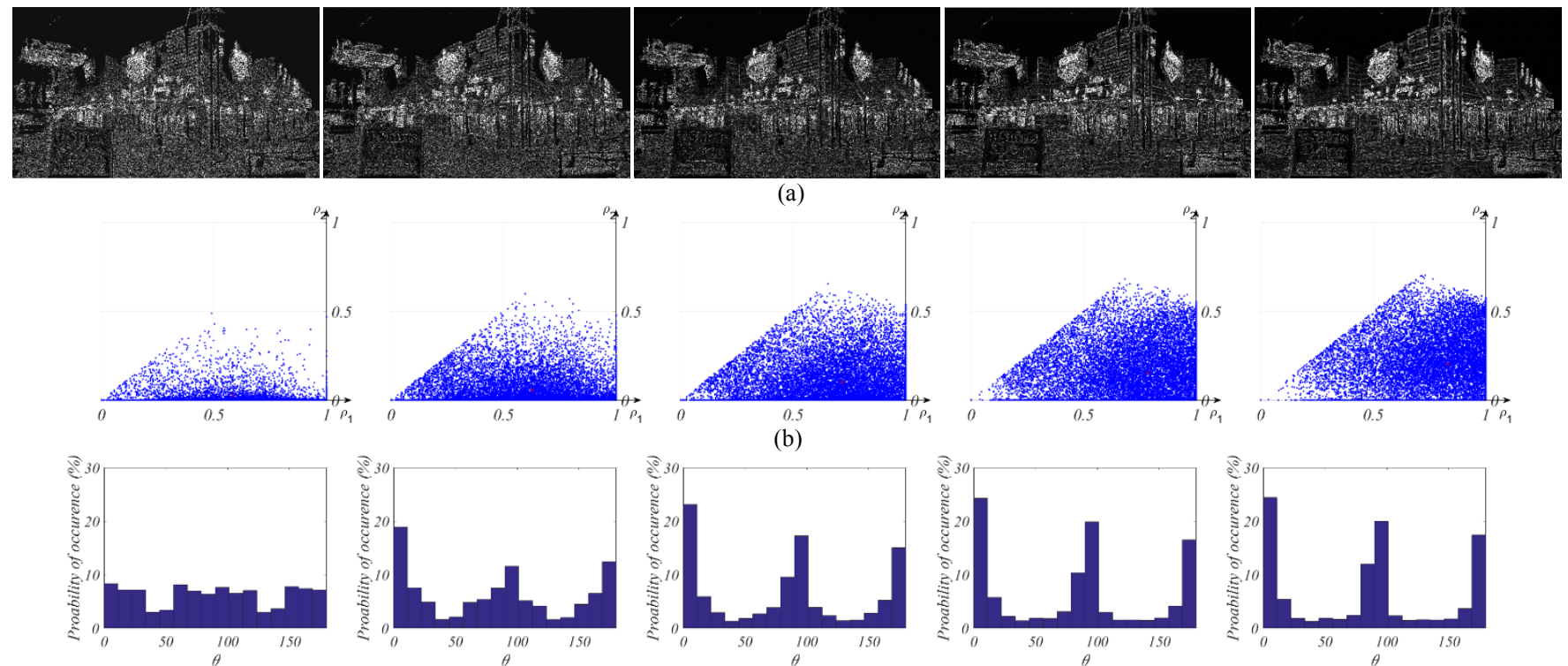

(c)

Fig. 4 (a) Residue images for "LasVegasStore" for separable model with different quantization parameters respectively QP = 17, 22, 27, 32, 37, (b) AutoCovariance analysis results for the obtained residues, (c) Histogram of estimated angles for generalized model

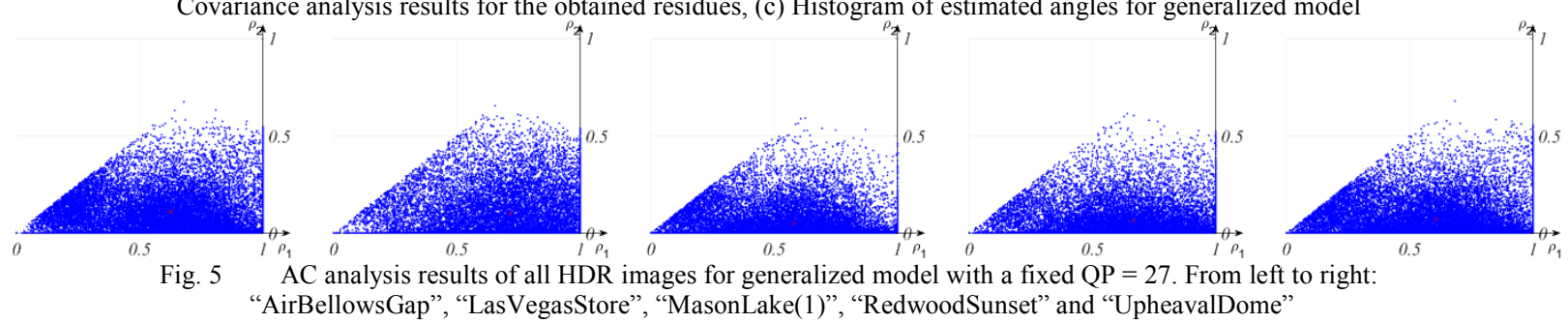

centroids of the scatter plots, indicated as the red cross on the figures. The scatter plots reveal that both the HDR images having the lowest (UpheavalDome) and the highest dynamic ranges (LasVegasStore) have sufficient number of blocks whose structure is dominated in one direction. Similarly, both the images having the lowest (AirBellowsGap) and highest spatial activity (MasonLake(1)) exhibit also directional structures in its blocks as indicated with the concentration along the $\rho_{1}$ direction. Overall, the covariance analysis results indicate that the HDR residuals have some 1-D characteristics and may be better compressed if 1-D transforms are used in addition to a 2-D transform.

\section{Proposed Residue Coding and Its Performance}

The proposed residue coding consists of two stages as the conversion to 8-bits and the coding of 8-bit images (Fig. 1).

\section{A. Conversion to 8-bits}

For the conversion of the residue signal to 8-bits, as the first method, we utilize the Sigmoid and Logit functions proposed in [11]. Due to the lack of space, the description is only given for the Sigmoid function here. The proposed algorithm [11] involves the following steps:

- The residue signal is first normalized to $[0,1]$ range with respect to its maximum and minimum values.

- The normalized residue denoted as $r_{n}$ is passed through the Sigmoid function:

$$
R_{n}=1 / 1+e^{-S\left(r_{n}-m\right)}
$$

- The resulting values are multiplied with 255 and rounded to obtain 8-bit image.

The $m$ parameter in the algorithm is taken as the arithmetic mean of $r_{n}$ values. The $s$ parameter is selected as the value which minimizes the mean square error (MSE) between the original residue and reconstructed residue signals after the conversion to and reconversion from 8-bits by applying an iteration over $s$ parameter recursively.

As a second content based solution for the 8-bit conversion, the optimum tone mapping of Mai [3] is adapted to the residue signal. The optimum tone mapping [3] minimizes the MSE between the logarithmic $\left(\log _{10}\right)$ values of the luminance of original HDR image and its reconstructed version. Given that the logarithm of the luminance of HDR image and the pixel values of tone mapped LDR version are denoted as $l$ and $v$ respectively, the tone mapping curve is first parameterized as a piece-wise linear function with the nodes $\left(l_{k}, v_{k}\right)$. Each segment $k$ between two nodes $\left(l_{k}, v_{k}\right)$ and $\left(l_{k+l}\right.$, $v_{k+1}$ ) has a constant width in HDR values equal to $\delta$. The tone mapping curve is then uniquely specified by a set of slopes,

$$
s_{k}=\left(v_{k+1}-v_{k}\right) / \delta .
$$

Mai et al. [3] eventually derived the $s_{k}$ values as 


$$
s_{k}=v_{\max } \cdot p_{k}^{1 / 3} / \delta \cdot \sum_{k=1}^{N} p_{k}^{1 / 3},
$$

where $p_{k}$ is the summation of the normalized histogram of luminance values for the $k$-th bin, $N$ is the total number of bins, and $\mathrm{v}_{\max }$ is the maximum LDR pixel value. The $\delta$ parameter in [3] is selected as 0.1 considering the sensitivity of human visual system (HVS) for the tone mapping in the base layer. Hereafter, we assume the $\delta$ parameter as a design parameter as the resulting residue mostly varies in the range of $[-0.1,0.1]$. Therefore, the $\delta$ value is iteratively changed and the one giving the minimum MSE between the original and reconstructed residue is chosen for the implementation.

\section{B. Standard and Directional Coding of the 8-bit Residue}

We compare the standard coding of the converted 8-bit residue images with the directional coding. Standard coding refers to coding with a standard H.264/AVC encoder, in particular the JM reference software [16]. Directional coding refers to a modified JM reference software encoder that uses several 1-D transforms in addition to the standard 2-D DCT transforms of the codec.

In particular, the directional codec used in our experiments is the modified H.264/AVC codec in [12]. It contains 8 1-D block transforms for $4 \times 4$ residue blocks and 16 1-D block transforms for $8 \times 8$ residue blocks. The 81 -D block transforms that are used for $4 \times 4$ blocks are shown in Fig. 6. Each arrow in the figure indicates a group of pixels on which a 1-D DCT is applied. The directions of the DCTs in the same block are roughly along the same direction. The directions of all 8 blocks roughly cover 180 degrees. The sixteen 1-D block transforms that are used for 8x8-pixel blocks are similar and can be seen in [12]. Note that other related compression modifications in the used directional codec, such as entropy coding of the 1-D transform coefficients with alternative scans, selection of the best 1-D transform or the 2-D DCT in each residue block, and signaling of the chosen transform in each residue block are all performed the same way as in [12].

Finally, note that while [12] uses the additional 1-D transforms to code inter prediction residue frames, this paper uses them to code HDR residue frames as intra-frames, similar to [17]. The coding procedure, however, remains the same. A block from the HDR residue is intra predicted, and the intra prediction residual block is coded with either a 1-D transform or the standard 2-D DCT, which is determined by the encoder with rate-distortion optimized transform selection.

\section{Comparison of Rate-Distortion Results}

In order to compare the performance of residue coding, the bitrate (QP) of the base layer is kept fixed and the residue signal is converted to 8-bits using two different methods and coded by using different QPs $(22,27,32,37)$. The distortion is computed in terms of the mean square error between the original and reconstructed HDR images (HDR-MSE), perceptually uniform peak signal to noise ratio (PU-PSNR) [18], and high dynamic range visible difference predictor (HDR-VDP) [19]. The bitrate is taken as the total bitrate of base layer and residue layer.

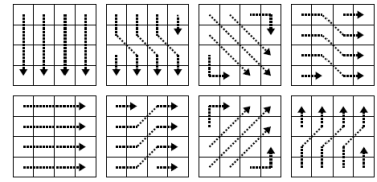

Fig. 6 The 8 1-D block transforms used in [12].

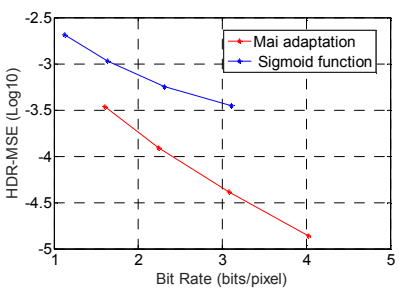

(a)

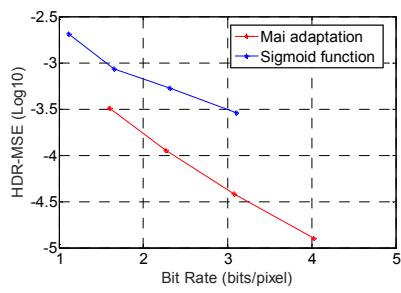

(b)
Fig. 7 HDR-MSE vs. Bit Rate (bits/pixel) curves for different 8-bit conversions for standard (a) and directional coding (b) for LasVegasStore.

Fig. 7 gives the rate distortion curves for the two compared 8-bit conversion methods, Sigmoid function and Mai adaptation, for standard [16] and directional coding [12] in (a) and (b), respectively, for LasVegasStore. The similar results are obtained for the other test images as well. The adaptation of the optimum tone mapping [3] to HDR residue significantly surpass the performance of the Sigmoid function for both the standard and directional coding with its content dependent characteristics. The gain in HDR-MSE for the same bitrate is about 0.3 to $0.9 \mathrm{~dB}$ for standard and directional coding. In the remaining experiments, Mai adaptation is used for the 8-bit conversion of the residue due to its better performance.

Fig. 8 (a, c, e, g and i) give the rate distortion curves in terms of PU-PSNR for the standard and directional coding for the utilized five HDR images. The directional coding gives better performance for all the images compared to standard coding. The gain in PU-PSNR is $0.2 \mathrm{~dB}$ to $0.6 \mathrm{~dB}$ depending on the bitrate and image. This corresponds to a gain in the bitrate of about 2-6\% for fixed PU-PSNR. The obtained results are in line with the reported gains for LDR coding residue in [17]. Note that the gain in the proposed method using Mai adaptation and directional coding compared to the baseline method using Sigmoid function and standard coding [11] is the cumulative gain obtained with 8-bit conversion and directional coding.

The comparisons are performed in Fig. 8 (b, d, f, h and j) in terms of HDR visible difference predictor (HDR-VDP). With HDR-VDP metric, directional codec is not consistently better than the standard codec. Although directional coding mainly improves coding of edges and detailed textured regions, it is not always possible to conclude that this improvement always produces better HDR-VDP results which can better model HVS perception.

\section{CONCLUSIONS}

We indicate that the HDR image coding residue obtained as the difference between the original and inverse tone mapped HDR images possesses directional characteristics for HDR images varying in terms of dynamic range and spatial activity. The directional characteristics of the residue have been found becoming more dominant as the quantization in 


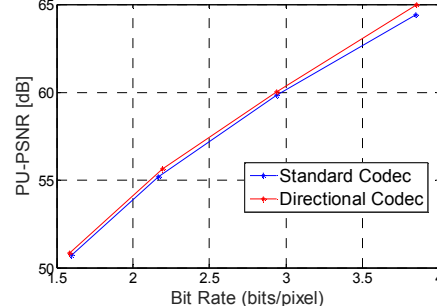

(a)

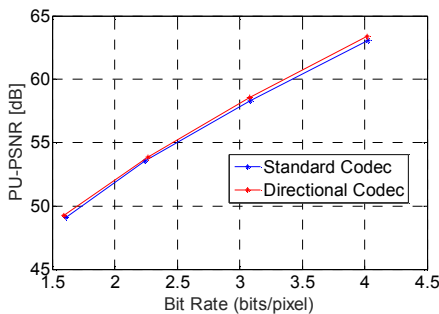

(c)

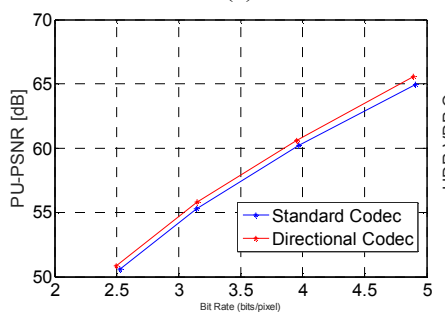

(e)

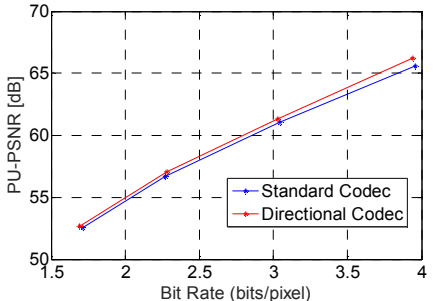

(g)

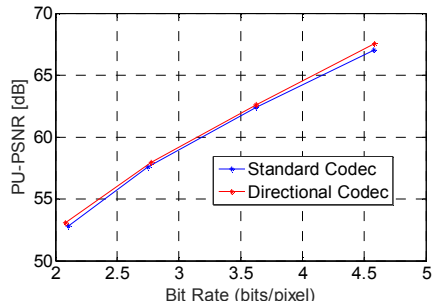

(i)

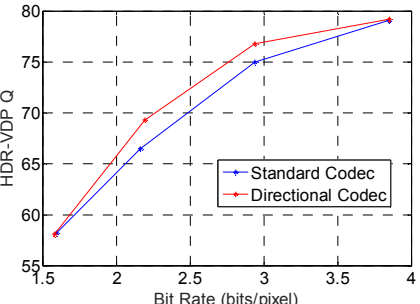

(b)

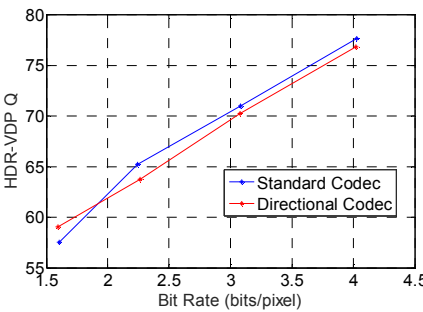

(d)

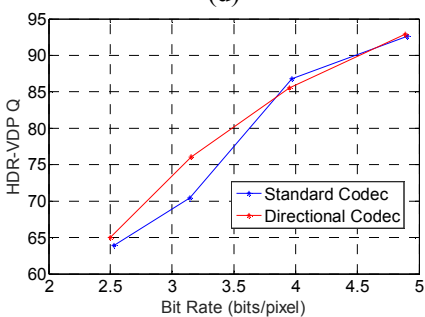

(f)

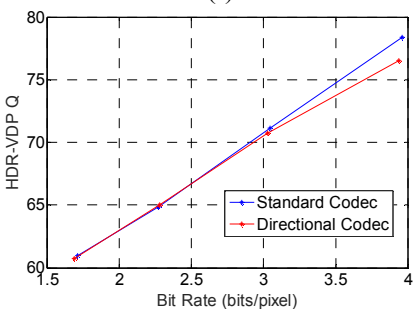

(h)

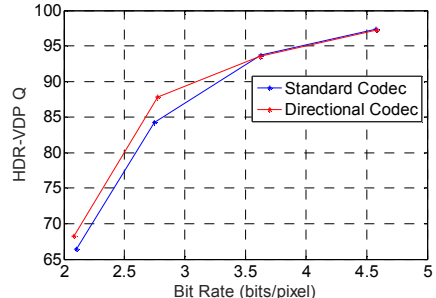

(j)
Fig 8. PU-PSNR vs. Bit Rate (bits/pixel) curves (left column) and HDR-VDP (Q) vs. Bit Rate (bits/pixel) curves (right column) for standard and directional coding for AirBellowsGap (a,b), LasVegasStore (c,d), MasonLake(1) (e,f), RedwoodSunset $(\mathrm{g}, \mathrm{h})$ and UpheavalDome $(\mathrm{i}, \mathrm{j})$. Mai adaptation is used for 8 bit conversion in both cases.

base layer increases. The proposed content dependent 8-bit conversion and directional coding method outperforms the existing function based 8-bit conversion and standard coding due to its verified directional characteristics.

\section{ACKNOWLEDGMENT}

This research is supported by The Scientific and Technical Research Council of Turkey (TÜBİTAK) and the French Ministry of Foreign Affairs under the common program BOSPHORUS with the project no 115E830.

\section{REFERENCES}

[1] Francesco Banterle, Alessandro Artusi, Kurt Debattista, and Alan Chalmers, Advanced High Dynamic Range Imaging, AK Peters (now CRC Press), 2011.

[2] E. François, C. Fogg, Y. He, X. Li, A. Luthra, and A. Segall, "High Dynamic Range and Wide Color Gamut Video Coding in HEVC: Status and Potential Future Enhancements", in Trans. on Circuits and Systems for Video Technology, Vol. 26, No. 1, pp. 63-75, Jan. 2016.

[3] Z. Mai, H. Mansour, R. Mantiuk, P. Nasiopoulos, R. Ward, and W. Heidrich, "Optimizing a Tone Curve for Backward-Compatible High Dynamic Range Image and Video Compression", IEEE Transactions on Image Processing, pp. 1558-1571, Vol. 20, No. 6, June 2011.

[4] R. Mantiuk, A. Efremov, K. Myszkowski, and H. P. Seidel, "Backward Compatible High Dynamic Range Mpeg Video Compression," $A C M$ Trans. Graphics (Proc. SIGGRAPH), Vol. 25, No. 3, pp. 713-723, 2006.

[5] A. Segall, "Scalable Coding of High Dynamic Range Video", Vol. 1, in Proc. IEEE ICIP, 2007.

[6] S. Liu, W. S. Kim, and A. Vetro, "Bit-depth Scalable Coding for High Dynamic Range Video" in Proc. SPIE Vis. Commun. Image Process. 2008, W. A. Pearlman, J. W.Woods, and L. Lu, Eds., 2008, Vol. 6822, p. 649212.

[7] A. Koz and F. Dufaux, "Methods for Improving the Tone Mapping for Backward Compatible High Dynamic Range Image and Video Coding", SP:IC, Vol. 29, Issue 2, pp. 274-292, Feb. 2014.

[8] D. Gommelet, A. Roumy, C. Guillemot, M. Ropert and J. LeTanou, "Rate-Distortion Optimization of a Tone Mapping with SDR Quality Constraint for Backward-Compatible High Dynamic Range Compression", in Proc. IEEE International Conference on Image Processing (ICIP2016), Arizona, USA.

[9] R. Boitard, R. Cozot, D. Thoreau, K. Bouatouch, "Motion-Guided Quantization for Video Tone Mapping", in Proc. IEEE International Conference on Multimedia and Expo (ICME2014), Chengdu, China.

[10] I. R. Khan, "A Nonlinear Quantization Scheme for Two Layer HDR Image Coding", Signal, Image and Video Processing, Vol. 10, Issue 5, pp. 921-926, July 2016.

[11] J. Mir, D. S. Talagala, H. K. Arachchi, and A. Fernando, "Adaptive Residual Mapping for an Efficient Extension Layer Coding in TwoLayer HDR Video Coding", in Proc. IEEE International Conference on Image Processing (ICIP2016), Arizona, USA.

[12] F. Kamisli and J.S. Lim, "1-D Transforms for the Motion Compensation Residual", IEEE Transactions on Image Processing, Vol. 20, No. 4, pp. 1036-1046, April 2011.

[13] Yehuda Dar and Alfred M. Bruckstein, "Motion-Compensated Coding and Frame Rate Up-Conversion: Models and Analysis", IEEE Transactions on Image Processing, Vol. 24, No. 7, pp. 2051-2066, April 2011.

[14] Harley Zhang and Jae S. Lim, "Analysis of One-Dimensional Transforms in Coding Motion Compensation Prediction Residuals for Video Applications," in Proc. of IEEE ICASSP, pp. 1229-1232, 2012.

[15] G. Valenzise, F. De Simone, P. Lauga, F. Dufaux, "Performance Evaluation of Objective Quality Metrics for HDR Image Compression", SPIE, Applications of Digital Image Processing XXXVII, San Diego, CA, August 2014.

[16] H.264/AVC JM Reference Software [Online]. Available: http://iphome.hhi.de/suehring/tml

[17] F. Kamisli, Transforms for Prediction Residuals in Video Coding, Ph.D. thesis, Massachusetts Institute of Technology, 2010.

[18] T.O.Aydin, R.Mantiuk, H.-P.Seidel, "Extending quality metrics to full luminance range images", Human Vision and Electronic Imaging XIII (Proceedings of SPIE) (2008) 6806-6810.

[19] R. Mantiuk, S. Daly, K. Myszkowski, H.-P. Seidel, "Predicting Visible Differences in High Dynamic Range Images - Model and its Calibration", in Proc. of Human Vision and Electronic Imaging X, IS\&T/SPIE's 17th Annual Symposium on Electronic Imaging 2005. pp. 204-214. 